\title{
TrainDy, a new UIC simulator for the longitudinal dynamics of trains
}

\author{
L. Cantone ${ }^{1}$, L. Müller ${ }^{2}$, D. Negretti ${ }^{3} \&$ V. Vullo ${ }^{1}$ \\ ${ }^{1}$ University of Rome "Tor Vergata", \\ Department of Mechanical Engineering, Italy \\ ${ }^{2}$ DB Systemtechnik (Deutsche Bahn AG), Germany \\ ${ }^{3}$ Faiveley Transport Italia, Turin, Italy
}

\begin{abstract}
In order to increase the productivity of freight trains and to harmonize the standards of different countries, there is the need for a new and reliable numerical simulator, since the in-line test campaigns are expensive and they should be reduced to a minimum amount. Furthermore, this simulator should easily be customized by each Railway Operator. In order to face these challenges, the UIC decided to improve and to validate the software TrainDy, and to deliver its source code to railway Operators, Research Centres and Universities that will join an ad hoc Consortium, led by the UIC. This paper gives the outlines of the numerical models implemented in TrainDy and shows some of the results of the validation process undertaken.
\end{abstract}

Keywords: longitudinal forces, improving freight trains productivity, modelling of pneumatic braking.

\section{Introduction}

Longitudinal forces (LF) exchanged by two consecutive vehicles of a train have a great impact on suitable length, applicable traction power, capacity and permissible speed of freight trains. Wrong decisions concerning these parameters result in accidents due to derailments, damage of wagons, goods and railways, but also in higher maintenance costs and lowered capacity. In order to increase freight train productivity, the International Union of Railways (UIC) has provided software for the calculation of LF for more than 25 years to the participating railways. Now the UIC has decided to develop a new system and to 
offer this system to a community that has the opportunity to participate in the development of this software due to an Open Source Solution. The new Train Dynamic Software called TrainDy [1] - developed by the University of Rome "Tor Vergata", with the financial support of Faiveley Transport of Italy - is programmed in MatLab and will be available to all members of a consortium led by the UIC. The software has already been subjected to a validation process by the UIC expert group and reached the UIC Certificate in April 2008. This process was divided into two main parts: pneumatic validation and dynamic validation. The pneumatic validation led to the mapping of the most widely used European braking devices. It provides a maximum error of $10 \%$, comparing the pressures in the braking cylinders versus the experimental data concerning inline trains. The necessary test run data were provided by three main European Railways Companies (Deutsche Bahn, SNCF and Trenitalia). Additionally Faiveley Transport provided experimental results of the their own train brake simulator. Dynamic validation was undertaken by matching the longitudinal forces and the stopping distances both with the software previously used by the UIC and, directly, versus the experimental data. The experimental test campaigns considered also addressed the study of the longitudinal forces for long freight trains with more than one braking locomotive (distributed braking). This paper shows some of the most significant steps and results of the whole validation process: once TrainDy will be fully delivered to the UIC in autumn 2008, the main Operators will have the possibility of easily agreeing on new inter-operable train configurations with enhanced productivity and the same level of safety. Moreover, they will directly access the code lines having the possibility of improving some features and/or of customizing some software characteristics.

\section{Numerical models}

For freight trains with pneumatic braking, in order to properly compute their longitudinal dynamics, it is mandatory to evaluate the pressure of the air in the brake pipe following any operation of the driver's brake valve. In this section, the main models implemented in TrainDy, in order to compute the longitudinal forces, will be provided.

\subsection{Pneumatic module}

The main brake pipe is modelled as a circular pipe with variable cross section (quasi 1D model) from which air can be blown in or spilled out; spilling out or blowing in can be performed from the head and/or the tail of the train, but also from more than one position in the middle of the train. In this way, it is possible to model an "end of train" device or distributed braking (i.e. more than one braking locomotive along the train). The spilling out of the air from the brake pipe is used to model, in a simplified way, the accelerating chambers of the control valves along with their auxiliary reservoirs, see Figure 1, where control valves and braking cylinders are skipped.

From the conservation of mass and energy and the balance of momentum within the above hypotheses the air governing equations become: 


$$
\left\{\begin{array}{l}
\frac{\partial \rho}{\partial t}+u \frac{\partial \rho}{\partial x}+\frac{\rho}{S} \frac{\partial(u S)}{\partial x}=-\frac{m}{S d x} \\
\frac{\partial u}{\partial t}+\frac{1}{\rho} \frac{\partial p}{\partial x}+u \frac{\partial u}{\partial x}=\frac{\tau}{D}+\frac{u}{\rho} \frac{\dot{m}}{S d x} \\
\frac{\partial q}{\partial t}+u\left(\frac{\partial q}{\partial x}+r \frac{\partial T}{\partial x}\right)+r \frac{T}{\rho S} \frac{\partial(\rho u S)}{\partial x}= \\
4 \frac{\phi_{T}}{\rho D}-\frac{\tau u}{D}-\frac{i t}{S d x} \frac{1}{\rho}\left[\left(c_{v}+r\right) T_{l}+\frac{1}{2} u_{z}^{2}-q\right]
\end{array}\right.
$$

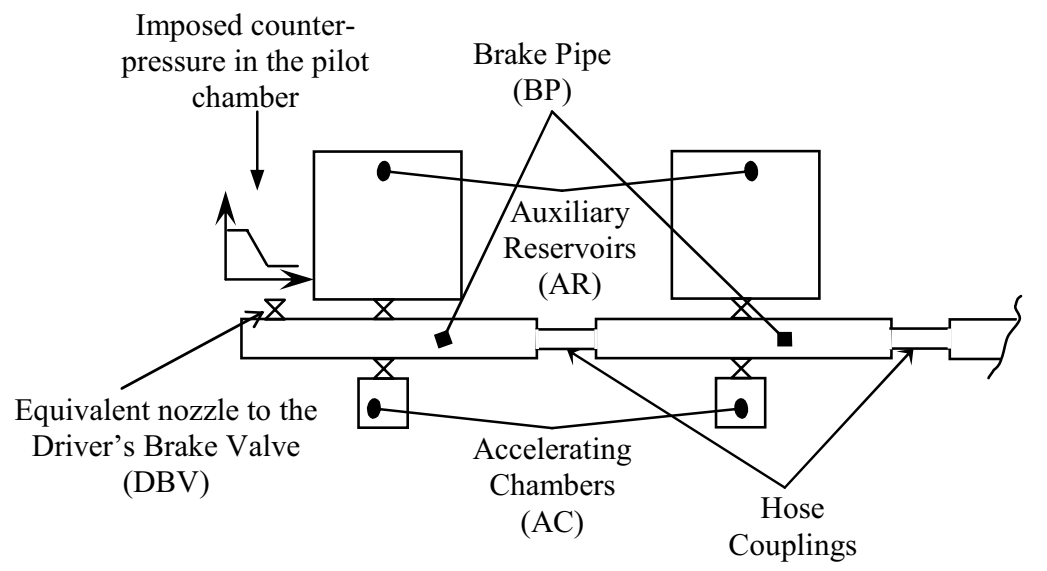

Figure 1: $\quad$ Sketch of the main devices modelled.

\subsubsection{Driver's brake valve}

The driver's brake valve is modelled as a nozzle with an equivalent diameter, tuned on experimental data. The determination of the mass flux $\dot{m}$, flowing through the equivalent cross-section, is obtained using the relation [2]:

$$
\dot{m}=C_{q} \cdot S_{L B V} \cdot C_{m} \cdot \frac{P_{u}}{\sqrt{T_{u}}},
$$

where $S_{D B V}$ is the equivalent nozzle cross-section, whereas the flow coefficient $C_{q}$ and the mass flow parameter $C_{m}$. Then the lateral flux speed used in (1) is given by:

$$
u_{i}=\frac{\dot{m}}{\rho_{d} \cdot S_{D B V}},
$$

where $\rho_{d}$ is the downstream air density in the equivalent model.

During a braking, the upstream pressure of the nozzle is the pressure of the brake pipe, while its downstream pressure is either the atmospheric pressure, for an emergency braking, or, for a service braking, the pressure of the pilot chamber of the driver's brake valve. In the latter case, the downstream pressure is time-variable, according to a law provided by the 
constructor of the valve. On the other hand, during a releasing, the upstream pressure of the nozzle is the pressure of the pilot chamber, yet provided by the valve constructor, whereas the downstream pressure is the pressure of the brake pipe. Since the actual pneumatic circuits used for the three previous manoeuvres are different, there are also three equivalent diameters of the nozzle that simulates the same driver's brake valve; it is worth to emphasize that these diameters need to be determined only one time and they do not depend on the length of the train.

\subsubsection{Accelerating chambers}

During a braking, the accelerating chambers of the control valves are modelled as lateral small volumes connected to the brake pipe by means of nozzles of equivalent constant diameters. The pressure of the air in these volumes changes according to the inflow flux mass, holding constant, for hypothesis, the temperature of the air in the volume of the accelerating chamber. In this case, the upstream pressure of the nozzle is the pressure in the brake pipe and the downstream pressure is the pressure of the air in the small volume; after a certain time period, the pressure of the air in the small volume is bigger than the pressure in the brake pipe and the air goes back, from the small volume to the brake pipe. Both the nozzle diameter and the capacity of the lateral volume are tuned on experimental measurements; it is worth to underline that this tuning occurs only one time and it neither depends on the manoeuvre (emergency or service braking) nor on the train length.

\subsubsection{Auxiliary reservoirs}

The auxiliary reservoirs are modelled as lateral big volumes connected to the brake pipe by means of a nozzle with variable diameter [1] and they communicate with the brake pipe only during a releasing. From the modelling point of view, the auxiliary reservoirs are similar to the accelerating chambers except that their initial pressure depends on the previous braking, because the air in the auxiliary reservoirs has been used to fill the braking cylinders.

\subsubsection{Control valve}

Once computed the pressure in the main brake pipe, the control valve transfer function along with the limiting curve of the braking cylinders are used in order to calculate the time evolution of the pressure in the braking cylinders. In the very beginning of the activation of the control valve, the filling or the emptying of the braking cylinders, respectively for braking and releasing, is performed only mathematically according to [3]; then, the pressure in the braking cylinders is controlled in time and depends, not only on the pressure in the brake pipe but also on the limiting curve.

\subsection{Dynamic module}

\subsubsection{Buffers/drawgears}

In its basic modelling, a train is regarded as series of masses connected by non linear springs, these springs have a behaviour as in Figure 2. When the relative 
speed of two consecutive wagons is in the interval among the loading and unloading speed, the exchanged force is computed by the following formula:

$$
F(x, v)=c(v) \cdot F_{\text {un-load }}(x)+[1-c(v)] \cdot F_{\text {load }}(x)
$$

The force-stroke characteristic can be provided in two different ways: a) giving the damping and some points of the loading curve; $b$ ) providing points both for loading and unloading curve; in both cases, the limiting speeds of loading and unloading must be given. TrainDy uses a cubic piecewise interpolation of the input data, which assures slope continuity, in order to improve numerical integration.

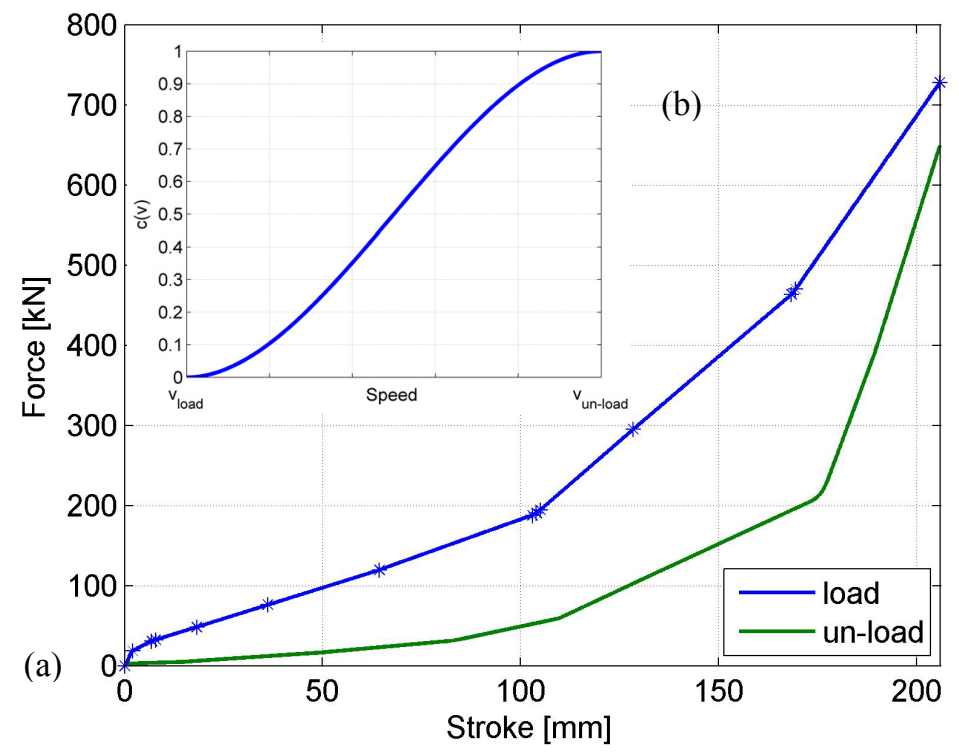

Figure 2: (a) Example of force-stroke characteristic; (b) smoothing curve.

\subsubsection{Friction coefficient wheel/braking shoe}

The laws of the friction coefficient implemented in TrainDy are the same described in [4]; moreover, a friction coefficient law, that considers the effects of specific pressure (among shoe and wheel) and of the running speed, has been implemented, considering Trenitalia experimental test data. During the validation process, the results in terms of stopping distance and of longitudinal forces using Trenitalia experimental law or the Karwatzki friction coefficient law were quite similar, in many cases.

\section{Validation}

The software has already been subjected to a validation process by the UIC expert group and reached the UIC Certificate in April 2008. This process was 
divided into two main parts: pneumatic validation and dynamic validation. The pneumatic validation led to the mapping of the most widely used European braking devices. It provides a maximum error of $10 \%$, comparing the pressures in the braking cylinders versus the experimental data concerning in-line trains. The needed test run data were provided by three main European Railways Companies (Deutsche Bahn, SNCF, Trenitalia). Additionally Faiveley Transport provided experimental results of the their own train brake simulator.

The TrainDy algorithms have been fully validated for the pneumatic part and dynamic part. The pneumatic validation took into account 28 simulations and test runs delivered by DB, SNCF and Trenitalia: 14 in service braking and 14 in emergency braking condition. The pneumatic module has been validated in traditional conditions (loco at the head of the train) and also in distributed braking conditions (more than one braking loco along the train). The dynamic validation was based on the previous test runs checking the stopping distance and the longitudinal forces.

In the following, for conciseness reasons, only two DB AG tests data comparisons are shown: TCS $12006 \# 003$ and Freight 1200017.

Figure 3 reports the pressure drop in the main brake pipe, for the test case TCS $12006 \# 003$ during an emergency braking from $24 \mathrm{~km} / \mathrm{h}$; the reported result refers to a braking with two driver's brake valves working (distributed braking) and when there is a time delay in braking activation of $4 \mathrm{~s}$. Focusing the attention on the wagon 56, it is evident that the first pressure drop is due to the emergency braking of the LOCO 1: the initial fast drop is due to the activation of the accelerating chamber, whereas the second part depends on the brake pipe emptying commanded by the valve on LOCO 1. After the driver's brake valve at

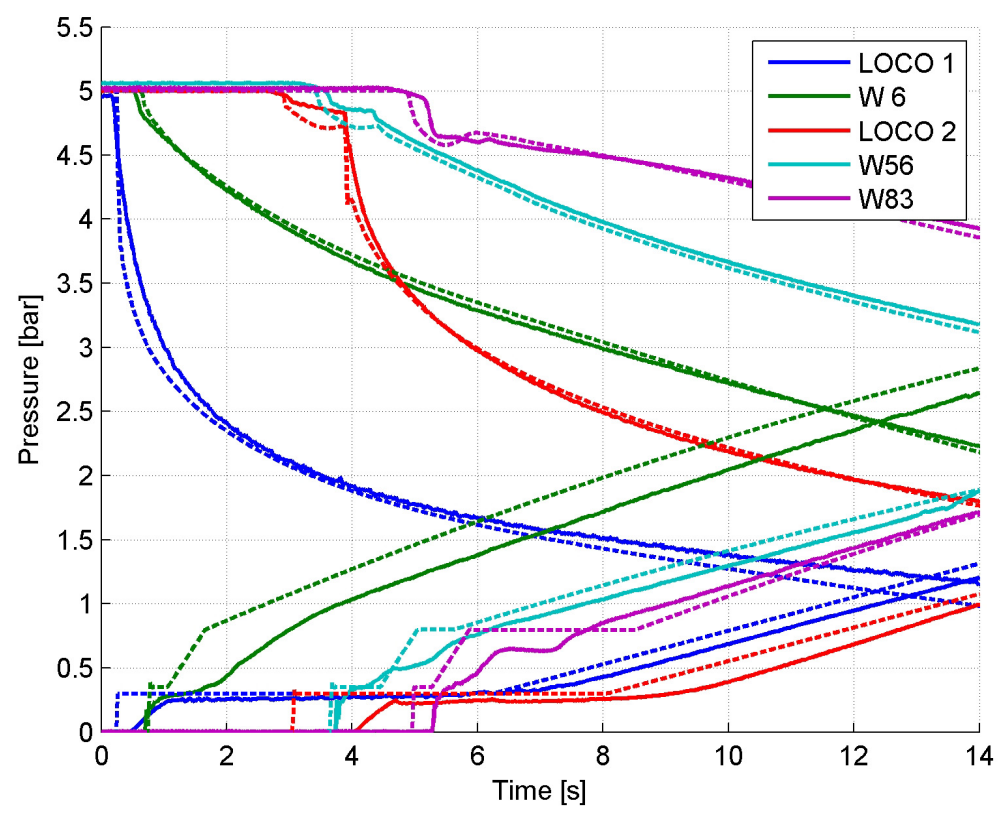

Figure 3: Emptying of the brake pipe and filling of the braking cylinders. 
LOCO 2 starts venting the air from the main brake pipe, the pressure drop of wagon 56 changes its slope.

This complex fluid-dynamic behaviour of the air is well caught by the simulator thanks to its refined, even if quasi mono-dimensional mathematical model of the main brake pipe. Figure 3 also shows the rising of the pressures in the corresponding braking cylinders: the agreement among the experimental and simulated results is considered satisfactory; as usual, there are minor differences at the beginning of the rising of the pressure, since TrainDy uses quasi-static transfer function and limiting curves to model control valves behaviour. Especially the initial experimental behaviour of wagon 83 is not well simulated by the numerical simulator. Anyway, note that, setting appropriate parameters of the in shot function, it is possible to reproduce the very strange behaviour of the two locomotives that start their pressure rising very late.

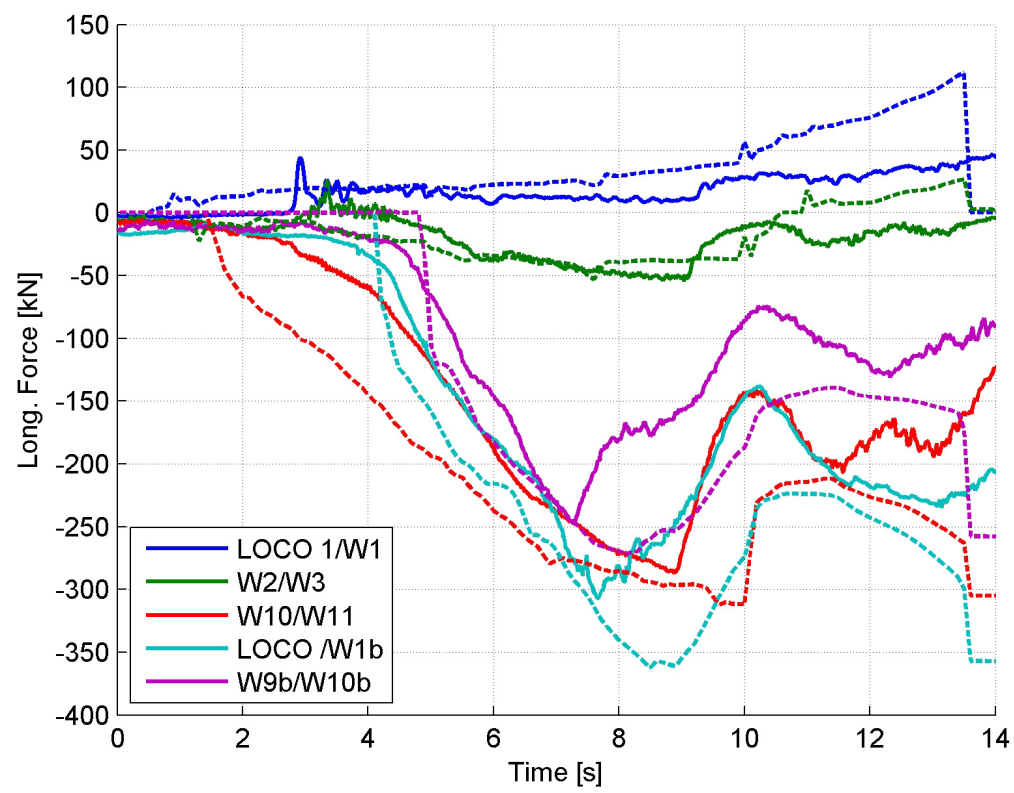

Figure 4: Longitudinal forces of TCS $12006 \# 003$.

Figure 4 shows the longitudinal forces for some couplings of the train. Of course there are some small differences among the simulated forces and the test runs: this happens because the numerical longitudinal forces are the result of the modelling of the pneumatic braking device, of the friction coefficient among wheels and shoes and of the force-stroke characteristics of the couplings. Moreover, it is worth to mention that the results have been obtained using the designed parameters, e.g. for the braked weights, but for sure in an experimental test the real parameters are always in a band around their nominal values and this, especially for long trains, can change the longitudinal forces. Anyway, the maximum relative difference among the maxima compression forces is around $20 \%$, a value that has to be considered satisfactory, also because there is a good matching on the shape of the numerical and measured longitudinal force. 


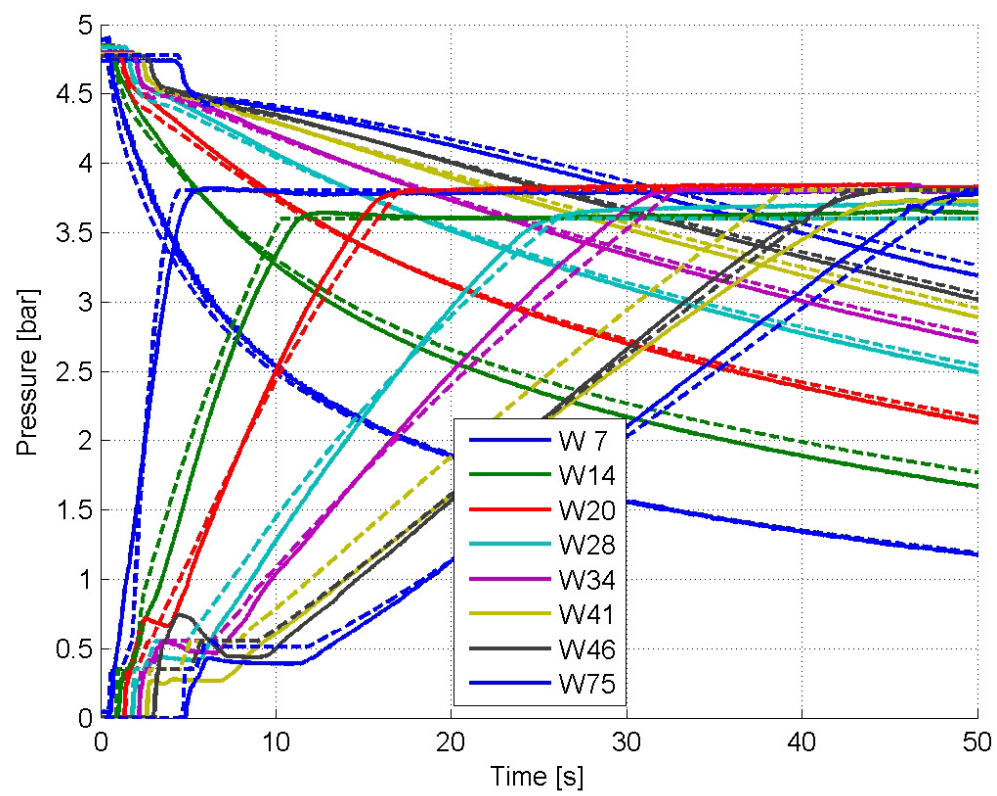

Figure 5: $\quad$ Pressure in brake pipe and braking cylinders.

The Freight test campaign of 2007 was dedicated to investigate the mass limits of classical train configurations, i.e. with only one locomotive or with two locomotives but with only one braking, for train length up to $1200 \mathrm{~m}$. The test 017 is an emergency braking from $100 \mathrm{~km} / \mathrm{h}$, in braking position "P" for both vehicles and locomotives, which were placed in front and at the end of the train.

Figure 5 shows the time evolution of pressure in brake pipe and in braking cylinders: the agreement among the experimental test data and the numerical counterpart is satisfactory. Also in this case, the major differences are located in the first phase of the filling of the braking cylinders when the numerical model of the control valve is not able to catch the actual behaviour of the air; anyway, Figure 5 clearly shows that the approximation is bordered in the first part of the filling when the pressures are low and when the consequences on the longitudinal forces are low. Another thing should be mentioned: Figure 5 clearly shows that the maximum pressure in the braking cylinder is reached, for each displayed wagon, when the pressure in the corresponding section of the brake pipe is around 3.2 bar: this means that the pressure in the braking cylinder is mainly driven by the transfer functions of the control valves; instead, this is not the case for the locomotives of Figure 3, where the first part of the filling of the braking cylinders is obtained by a particular shape of the limiting curve of the corresponding control valves and the maximum pressure value is reached when the pressure in the corresponding sections of the brake pipe is quite below 3.2 bar.

Figure 6 displays the speed versus running speed both for the test run and the numerical simulation: the achieved error on the stopping distance is quite low 


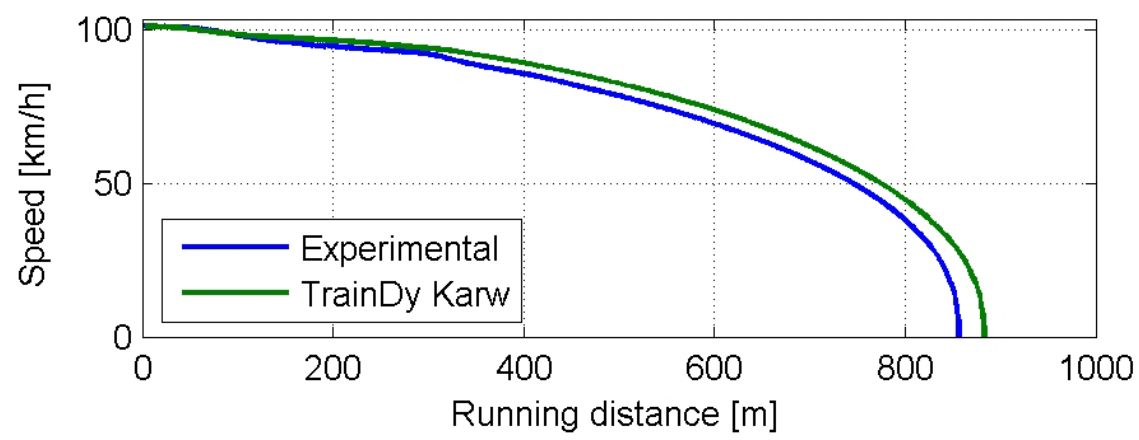

Figure 6: $\quad$ Speed versus running distance.

and around 3\%; the simulation has been carried out using the Karwatzki friction law providing also a good agreement on the shape of the two curves.

Finally, Figure 7 shows the comparison among he longitudinal forces for some train couplings of Freight 2007 017. The numerical and measured longitudinal forces closely agree even if the measured forces show some couplings with cut measured signal. In this test, in order to reduce the maximum compression forces, the loco at the head of the train does not brake and this reflects in tension force for the first couplings of the train (see coupling 7 in Figure 7).

\section{Conclusion and perspectives}

Longitudinal forces have a great impact on suitable length, applicable traction power, capacity and permissible speed of freight trains. Finally LF are responsible for higher transportation costs and unsafe train runs, if their impact was not investigated before establishing new train configurations or operational procedures.

The simulation of longitudinal forces and operational modes is the best and cheapest possibility to solve the above mentioned tasks, if the system delivers accurate and reliable results. The validation process of TrainDy demonstrated both, the correct physical basis of the system and the resulting accuracy. The approach to define standard values for general simulations representing an average of the parameter range of one vehicle type and to use specific parameters for one specific test run where all parameters are well known shows the possibilities of adapting different situations with one system.

This offers different perspectives for Railway Operators and vehicle/equipment constructors. Railway Operators are able to predict the level of forces for different kinds operation (brake regime), increasing velocity, higher loads and longer trains. The Railway industry is able to implement their own components, predict the impact on different above mentioned operational modes and to demonstrate the effect using a certified system.

Additionally research institutions are invited to enhance the source code. The UIC will establish a TrainDy Consortium with complete access to the source code of the system. The programming language MatLab assures the ability of 


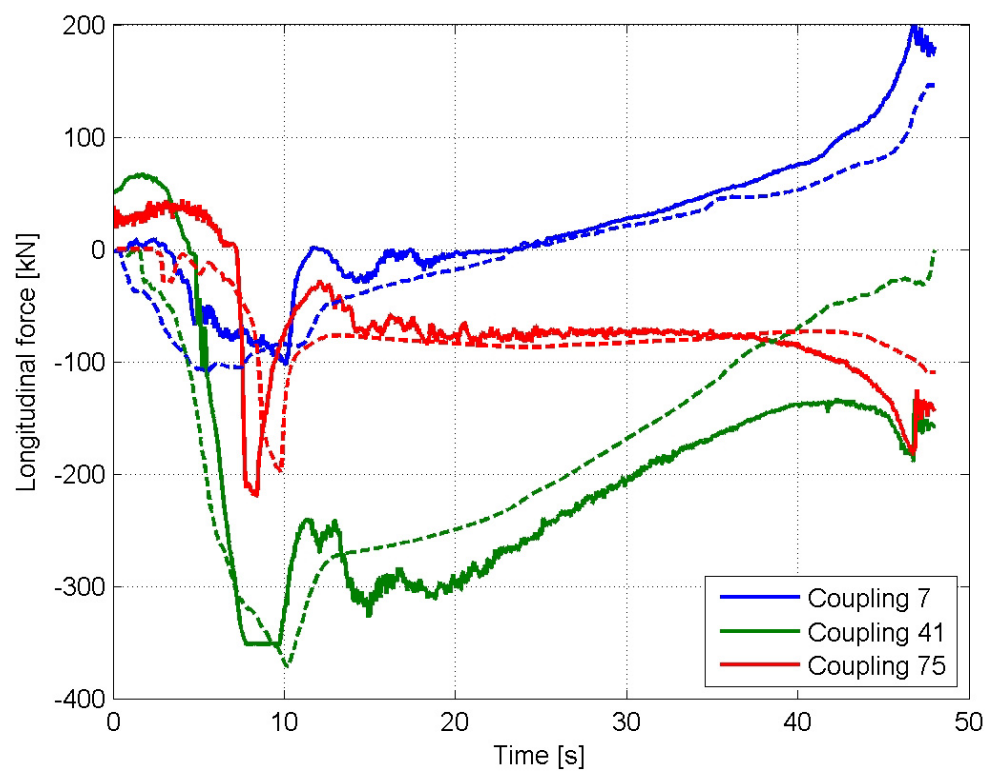

Figure 7: Comparison of the longitudinal forces.

specialists worldwide to understand the code and to modify it. To support the evolution of the system, research institutions get special conditions joining the consortium. The vision of the project is to motivate international research institutions to modify the source code and to adapt it to the regional requirements. The results of these enhancements are controlled by an expert team of the UIC, which certifies the official version. As a consequence the software has the potential to be used by a wide array of users and to develop faster than software without source code access.

After the final validation until October 2008, the UIC will immediately start to use the system to define new limits for international freight trains and to introduce these values in the corresponding UIC Leaflet 421. In parallel the UIC starts to build up the consortium.

\section{References}

[1] L. Cantone, E. Crescentini, P. Salvini, R. Verzicco, V. Vullo, "Modello numerico per lo studio della manovra di sfrenatura". INGEGNERIA FERROVIARIA. 2007, vol. 7/8, pp. 575-588.

[2] D. McCloy, H. Martin: "Control of Fluid Power: Analysis and design", $2^{\text {nd }}$ edition. Ellis Horwood Limited 1980

[3] UIC Leaflet 540-0 "Freins a air comprimé pour trains de marchandises et trains de voyageurs"

[4] T. Witt, "Integrierte Zugdynamiksimulation für den modernen Güterzug", Dissertation Institut für Verkehrswesen, Eisenbahnbau und -betrieb, Universität Hannover, Hannover 2005. 